\section{Molecular switches for regulating therapeutic genes}

Viral vectors have made it possible to deliver foreign genes to cells and tissues efficiently, stably, and apparently safely. These vector systems have made gene therapy a realistic possibility, and helped to carry many protocols into the clinical arena, with more than 300 phase 1 or 2 clinical trials currently underway (reviewed in Refs 1 and 2). Adeno-associated viral vectors (AAV) and adenoviruses have been used successfully in animals to treat disease aspects of numerous conditions including but not necessarily limited to hypertension, myocardial ischaemia, neuronal ischaemia, muscular dystrophy, arthritis, anaemia, haemophilia, cystic fibrosis, obesity and various types of cancer. Newer vectors including those based on lentivirus (HIV) are also showing promise. The elimination of viral genes from these vectors and the use of species-compatible transgenes have helped to neutralize host rejection and allow stable transmission and retention of the genes. AAVs and lentivirus integrate into the host genome and are potentially permanent; adenoviral vectors remain extrachromosomal and gene delivery is correspondingly less stable. Both situations may have benefits.

Until recently the most common strategy has been to express the transgene from a strong promoter, such as cytomegalovirus (CMV). This allows maximal expression of the transgene, but the expression is constitutive and unrestricted in the infected host tissues. In many cases it is desirable to restrict the transgene expression to a specific cell or tissue type. Tissue-specific promoters incorporated in place of the ubiquitous promoters can convey faithful tissue specificity within the viral vectors. Targeting of viral vectors to endothelial cells, brain, cardiac muscle, smooth muscle, skeletal muscle, liver, kidney, retina and certain tumours using tissue-specific promoters has been reported. ${ }^{1,2}$ The use of tissue-specific viral vectors is likely to increase.

While the use of tissue-specific promoters confers restricted expression, and thence tissue targeting, there still remains the requirement to regulate this expression appropriately within the tissue. In general terms, appropriate regulation means the capability to turn the transgene on or off in response to symptoms (expression) of the targeted disease. A number of elegant strategies have been shown to accomplish this; they all make use of inducible elements, and factors that can be rapidly regulated by the (oral) administration of a second-step drug. The first such system to be exploited employed elements regulated by the antibiotic tetracycline. Appropriate incorporation of these elements into transgene promoters has allowed sustained, tissue-specific expression of Ad and AAV vectors that can be regulated by tetracycline. ${ }^{3}$ Two additional procedures, based on the same principle, subsequently reported equivalent or even tighter regulation than the tetracycline system. ${ }^{4,5}$ These reports demonstrated that it is possible to deliver a putative therapeutic gene to specific tissues in a completely dormant state. Expression could be induced at will by the oral administration of a second drug (rapamycin or mifepristone), that directs the formation of an active transcription factor complex on the otherwise silent promoter of the transferred gene. Tests on rhesus monkeys demonstrated the stable expression of a constitutive AAVerythropoietin vector in muscle for more than 1 year, and of the regulatable AAV-erythropoietin for at least 2 months (decreased stability of the regulated AAV was attributed to immune reaction of epitopes on the activating transcription factors). The value of these results lies not only in the potential for strict and rapid control of therapeutic transgenes by exogenous drugs (that lack detectable side-effects), but they also confirm the stability of integrated AAV vectors.

The availability of stable, efficient, tissue-specific and tightly regulated vectors will have a significant positive impact on the future of gene therapy. These should be applicable to numerous human conditions that will respond to augmentation, substitution, or even inhibition of a specific gene product. One possible limitation of the method in some instances may be the requirement for exogenous regulation through the second drug, which will require additional monitoring and testing to determine the appropriate regulation of the transgene. There may be conditions when the treatment would be improved by a more direct communication between the disease and the therapeutic gene. Work from several groups including our own, suggest that it may be possible in some instances to circumvent the second step of exogenous regulation and activate the dormant transgene directly by an endogenous symptom of the targeted disease. This will be possible under conditions when a disease is stringently linked to a molecular phenotype that can directly impact gene expression. I will discuss below the possibility that hypoxia may be such an endogenous signal that could replace exogenous regulation in the gene-based treatment of select diseases such as myocardial ischaemia, stroke and diabetic retinopathy.

Cells and tissues respond to an oxygen deficit (hypoxia) by rapidly, and in some instances, strongly inducing a specific subset of adaptive genes that control key activities (reviewed in Ref. 6). Genes involved in 
anaerobic energy production, erythrogenesis and angiogenesis are induced to sustain energy levels, stimulate red blood cell production and generate more vessels. These genes may be induced very rapidly, within minutes after the cells become hypoxic. The rapid induction is mediated through the increased activity of one or more specific transcription factors that are highly sensitive to oxygen tension. One such factor called hypoxiainducible factor 1 (HIF-1) is undetectable in aerobic cells but accumulates within minutes after the cells are made hypoxic. The rapid appearance of HIF-1 is possible because HIF-1 is synthesized continuously in mammalian cells. It is rapidly ubiquitinated and degraded under conditions of normal oxygenation, but degradation is blocked and HIF-1 accumulates immediately under hypoxia. Conversely, when hypoxic cells are reoxygenated, HIF-1 is again degraded and disappears within minutes. The rapid kinetics of these responses are compatible with a possible role for the HIF-1 pathway in the regulation of integrated vectors containing therapeutic genes. A second compatible feature is the high specificity of gene regulation by hypoxia; HIF-1 binds to and activates only genes that have a consensus site containing the sequence element ACGTG and compatible flanking sequences (known collectively as hypoxia responsive enhancers, or HREs).

Could tissue hypoxia be used to regulate the expression of a therapeutic gene and replace the exogenous second step regulation? The feasibility of such a procedure must be considered within the context of specific target diseases. The most suitable candidate diseases are ischaemia-related, where blood flow to a region of tissue is restricted or eliminated and hypoxia is a central and consistent feature of the disease. This disease category includes, although is not necessarily limited to myocardial ischaemia, stroke, peripheral (limb) ischaemia, diabetic retinopathy and solid tumours. In all of these conditions the effected tissues are subjected to ischaemia and hypoxia that may be intermittent or sustained. A number of criteria need to be met for ischaemia/hypoxia to be a viable endogenous signal to regulate transferred foreign genes. These include: (1) efficient delivery of a tissue-specific vector to the target tissue; (2) long-term stability and viability of expression; (3) low expression of the transferred gene under aerobic (uninduced) conditions; and (4) strong and rapid activation of the gene by (patho)physiological ischaemia or hypoxia in vizo.

The first three criteria are largely satisfied by the demonstration, discussed above, that recombinant, tissue-targeted AAVs remain responsive to exogenous activation, and express the transferred gene several months after delivery. ${ }^{4}$ This confirms previous work that has demonstrated efficient delivery of genetic material to the myocardium, brain and vasculature using AAV, adenovirus, and Herpes simplex viral vectors, and while there are likely to be improvements in gene delivery techniques in the future, we can conclude that criteria 1-3 can be satisfactorily met.

Fulfillment of the fourth criterion involves properties of both the hypoxia-regulated vector and the target tissue. The vector containing the therapeutic gene is required to have low expression in normal (normoxic) tissue and be rapidly activated after the onset of ischaemia. The molecular genetic responses to hypoxia and ischaemia in vitro and in vivo indicate that this criterion

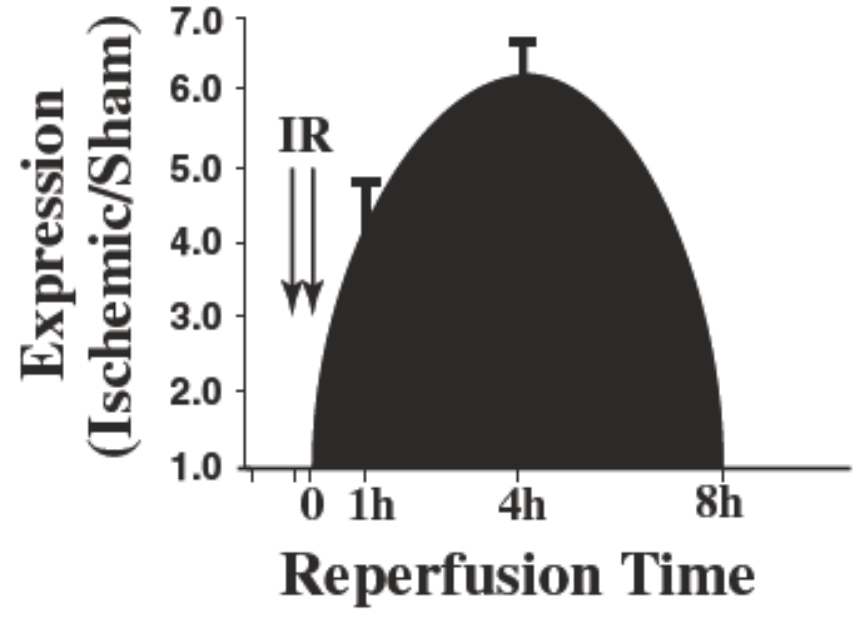

Figure 1 Trangrene activation in the rabbit left ventricle by 15 min of ischatemia.

will also be met. Although the magnitude and kinetics of the molecular genetic response to hypoxia is gene-specific, the threshold for hypoxia-mediated gene activation appears to be consistent. Most HRE pathways are fully activated by exposing cultured cells to $2-5 \% \mathrm{O}_{2}$, and these pathways are dormant at higher $\mathrm{O}_{2}$ tensions. The oxygen sensors are not known but they may be in equilibrium with intermediates of bioenergetic pathways, such as the heme moieties of cytochromes, or other hemecontaining complexes. ${ }^{6}$ Pathophysiological oxygen tensions resulting from ischaemia are likely to drop rapidly to the hypoxia-activation threshold. This is shown by the rapid activation of endogenous HRE-regulated genes in hypoxic or ischaemic tissues. The level of induced gene expression is determined by the characteristics of the individual hypoxia-responsive promoter and ranges from a low three- to four-fold induction of some glycolytic enzyme promoters, through a median of $>10$-fold induction of promoters with more active, or multiple copy HREs, to the more than 100-fold activation of the endogenous erythropoietin gene. Therefore there is little doubt that signals from hypoxic/ischaemic tissues are sufficient to exert strong regulation over gene promoters that contain an appropriate arrangement of HREs.

In experiments carried out in this laboratory we tested the kinetics of the hypoxia/ischaemia-regulatory system directly in the context of myocardial ischaemia. ${ }^{7}$ Rabbit left ventricles were injected with a vector containing the luciferase gene linked to a minimal heart-specific promoter containing four tandem HRE repeats. After 1 week the same left ventricles were subjected to a single $15-\mathrm{min}$ period of ischaemia by ligating the coronary artery, and luciferase activity was measured at intervals after reperfusion. The results of these experiments are illustrated in Figure 1. The short ischaemic episode, indicated by the arrows, initiated a rapid activation of the transferred gene that was close to maximum after $1 \mathrm{~h}$ of reperfusion, peaked within $4 \mathrm{~h}$ and returned to base within $8 \mathrm{~h}$. In parallel experiments, there was no change in the expression of a control vector lacking the HREs (see Ref. $7)$. In these experiments we used a medium strength HRE 
combination that produced a six-fold maximum induction. More powerful HRE combinations are capable of at least 25 -fold activation over basal expression (K Webster et al, unpublished).

These results support the feasibility of a hypoxia/ischaemia-regulatory system that, in principal, could replace exogenous regulators of gene therapy vectors. The system would be applicable to the treatment of conditions in which ischaemia and/or hypoxia are integral features of the targeted disease. The dual regulation provides for tissue-specific transgene expression that is restricted physically and temporally to diseased (ischaemic) tissue. A specific advantage of such a regulation is the inherently rapid response that will result when the medication is regulated directly by the disease. This may be particularly important in the treatment of diseases such as myocardial ischaemia and stroke where the window of opportunity for treatment is small. In acute stroke patients, only tissue plasminogen activator (tPA) has been proven to provide neurologic improvement, and only when it is administered within $3 \mathrm{~h}$ of the stroke. ${ }^{8}$ Other neuroprotective agents such as anti-oxidants and the anti-apoptotic protein $\mathrm{Bcl} 2$ are considered promising candidates, again if they can be administered soon enough. Similarly, candidates for the treatment of myocardial ischaemia include anti-oxidants, anti-apoptotic gene products, vasodilators, and thrombolitics. As with stroke, early treatment of myocardial ischaemia is a critical parameter. It is not difficult to visualise how both of these conditions would benefit from a therapeutic gene product that is synthesized and released as soon as the symptoms of ischaemia appear.

Another potential application is the hypoxia-regulated delivery of pro-angiogenic genes to ischaemic tissues to promote revascularisation. There are currently several clinical trials in progress to determine the therapeutic potential of directly injected VEGF and basic fibroblast growth factor genes and proteins to stimulate angiogen- esis in ischaemic myocardium and ischaemic limbs (see Ref. 9 and references therein). Delivery of these factors by tissue-specific hypoxia-regulated vectors may be a more efficient and safer way to accomplish this goal, avoiding possible spillover of the viral transgene to other areas where increased angiogenesis is not desired.

\section{Acknowledgements}

This work was supported by NIH grant HL44578.

$$
\begin{array}{r}
K A \text { Webster } \\
\text { Department of Molecular and Cellular Pharmacology } \\
\text { University of Miami Medical Center } \\
\text { RMSB 6038, 1600 NW 10th Avenue } \\
\text { Miami, FL 33101, USA } \\
\text { Email: kwebster@chroma.med.miami,edu }
\end{array}
$$

\section{References}

1 Palu G, Bonaguro R, Marcello A. In pursuit of new developments for gene therapy of human diseases. J Biotechnol 1999; 68: 1-13.

2 Robbins PD, Ghivizzani SC. Viral vectors for gene therapy. Phar macol Ther 1998; 80: 35-47.

3 Ghersa $\mathrm{P}$ et al. Highly controlled gene expression using combinations of a tissue-specific promoter, recombinant adenovirus and a tetracycline-regulatable transcription factor. Gene Thenapy 1998; 5: 1213-1220.

4 Ye $\mathrm{X}$ et al. Regulated delivery of therapeutic proteins after in vivo somatic cell gene transfer. Science 1999; 283: 88-91.

5 Burcin MM. Adenovirus-mediated regulable target gene expression in vito. Proc Natl Acad Sci USA 1999; 96: 355-360.

6 Bunn HF, Poyton RO. Oxygen sensing and molecular adaptation to hypoxia. Physiol Rev 1996; 76:839-885.

7 Prentice $\mathrm{H}$ et al. Regulated expression of a foreign gene targeted to the ischaemic myocardium. Card Res 1997; 35: 567-574.

8 Zivin JA. Factors determining the therapeutic window for stroke. Neurology 1998; 50: 599-603.

9 Losordo DW et al. Gene therapy for myocardial ischaemia. Circulation 1998; 98: 2800-2804. 